\title{
NHERF1/EBP50 controls lactation by establishing basal membrane polarity complexes with prolactin receptor
}

\author{
FC Morales ${ }^{1,4}$, Y Hayashi $^{1,4}$, CS van Pelt ${ }^{2}$ and M-M Georgescu* ${ }^{\star, 1,3}$
}

The development of the lactating mammary gland is a complex multifactorial process occurring in mammals during pregnancy. We show here that this process requires NHERF1/EBP50 ( $\mathrm{Na/H}$ exchanger regulatory factor 1/ERM-binding phosphoprotein 50) expression and that successful lactation depends on NHERF1 allele copy number, with rates of 50 and $20 \%$ in $N H E R F 1(+I-)$ and $(-I-)$ mice, respectively. The prolactin receptor (PRLR)-STAT5 signaling provides the central axis triggering the differentiation of secretory mammary alveolar cells. In successfully lactating glands, NHERF1 is massively upregulated and forms complexes with PRLR, but also with $\beta$-catenin, E-cadherin and ezrin at the alveolar basal membrane, establishing basal polarity. In NHERF1-deficient glands, the basal polarity is disrupted, the PRLR levels and basal membrane localization are abolished, and the downstream STAT5 activation collapses with consequent reduction of milk protein synthesis. NHERF1/ EBP50, a protein deregulated in breast cancer, thus emerges as an important physiological mediator of milk secretion, by engagement of PRLR in multimeric complexes at the alveolar basal membrane with subsequent network activation leading to cell differentiation.

Cell Death and Disease (2012) 3, e391; doi:10.1038/cddis.2012.131; published online 20 September 2012

Subject Category: Cancer

In mammals, the ability of the mammary gland to secrete milk is absolutely essential for the survival of the newborns. During pregnancy, a whole program of cell proliferation and differentiation is set in place in the mammary gland that transforms its ductal architecture from the non-pregnant period into the lobulo-alveolar milk-secreting gland at the end of gestation. ${ }^{1}$ This program is triggered by the secretion of placental and pituitary hormones, the most important of which being progesterone and prolactin/placental lactogens. Whereas progesterone binds to a nuclear receptor to directly activate gene transcription and to promote mainly the proliferative expansion of the alveolar compartment, prolactin and placental lactogens are related peptides that bind to the prolactin receptor (PRLR) on mammary epithelial cells to induce both proliferation and differentiation of milk-secreting alveolar cells. PRLR is a one-pass transmembrane type I cytokine receptor. Ligand binding leads to PRLR dimerization and phosphorylation of docking tyrosine residues by the receptor-associated JAK2 kinase. ${ }^{2}$ Phospho-tyrosines situated near the carboxyl terminus (C terminus) of PRLR intracellular domain dock the STAT5A-B transcription factors via their $\mathrm{SH} 2$ domain and expose them to phosphorylation by
JAK2. Phosphorylated STAT5 dimerizes and translocates to the nucleus where it directly activates the transcription of molecules involved in the proliferation and differentiation of alveolar cells, including connexin-26, ${ }^{3}$ RANK-L ${ }^{4}$ and cyclin D1. ${ }^{5}$ STAT5 has also as direct targets the genes encoding the milk proteins, which define the mature state of the differentiated secreting alveolar cell. ${ }^{1}$

The importance of the central PRLR-STAT5 signaling axis for the development of the secretory gland has been elucidated in mice by knocking out the genes encoding the components of the pathway. Mice deficient for PRLR, ${ }^{6}$ JAK-2, ${ }^{7,8}$ STAT5A, ${ }^{9}$ cyclin D $1,{ }^{10,11}$ connexin-26, ${ }^{12}$ RANK-L and its receptor RANK ${ }^{13}$ fail to develop functional secreting alveoli during pregnancy and therefore fail to lactate their newborn.

We generated NHERF1-deficient mice ${ }^{14}$ and observed lactation defects reminiscent of those from mice deficient for the components of the PRLR-STAT5 pathway. NHERF1/ EBP50 (Na/H exchanger regulatory factor 1/ERM-binding phosphoprotein 50) contains two N-terminal PDZ (PSD95Dlg-ZO1) domains and a C-terminal ezrin-radixin-moesin (ERM)-binding region. ${ }^{15,16}$ Through its PDZ domains, NHERF1 interacts with a multitude of ligands that contain

\footnotetext{
${ }^{1}$ Department of Neuro-Oncology, The University of Texas MD Anderson Cancer Center, Houston, TX 77030, USA; ${ }^{2}$ Department of Veterinary Medicine, The University of Texas MD Anderson Cancer Center, Houston, TX 77030, USA and ${ }^{3}$ Department of Pathology, University of Texas Southwestern Medical Center, Dallas, TX 75390, USA

${ }^{*}$ Corresponding author: M-M Georgescu, Department of Pathology, The University of Texas Southwestern Medical Center, 5323 Harry Hines Boulevard, Dallas, TX 75390, USA. Tel: 214648 7174; Fax: 214648 6323; E-mail: maria-magdalena.georgescu@ phhs.org

${ }^{4}$ These authors contributed equally to this work.

Keywords: mammary gland development; lactation; epithelial polarity; NHERF1/EBP50; PRLR; STAT5

Abbreviation: BrdU, 5-bromo-2'-deoxyuridine; C, carboxyl; EBP50, ERM-binding phosphoprotein 50; ERM, ezrin-radixin-moesin; GST, glutathione S-transferase; IF, immunofluorescence; NHERF1, Na/H exchanger regulatory factor 1; MEFs, mouse embryonic fibroblasts; PDZ, PSD95-Dlg-ZO1; PRLR, prolactin receptor; WAP, whey acidic protein

Received 11.7.12; revised 03.8.12; accepted 08.8.12; Edited by G Raschella'
} 
specific PDZ motifs in their $\mathrm{C}$ terminus, ${ }^{17}$ whereas the ERMbinding region connects to the FERM domain of the ERM proteins. NHERF1 is present in a variety of cells and tissues, mostly localized at the plasma membrane. ${ }^{18-20}$ In the nonlactating mammary gland, NHERF1 has been detected apically in normal duct epithelial cells. ${ }^{20-22}$ We found that in milk-secreting alveolar cells, NHERF1 interacts and colocalizes with PRLR at the basal membrane and organizes multimeric complexes that maintain the basal membrane polarity and distribution of PRLR. In NHERF1-deficient animals, the architecture of the basal membrane of alveolar cells is disrupted, leading to PRLR depletion, abolished STAT5 signaling and defective cell differentiation. These findings reveal NHERF1 as a PRLR regulatory protein, with important implications for the differentiation and function of the lactating mammary gland.

\section{Results}

Severe nursing deficit in NHERF1-mutant mice leads to significant neonate mortality and decreased offspring weight. Confronted with a severe breeding deficiency of the NHERF1-mutant mice, we examined in a prospective study the age of NHERF1-mutant females at their first pregnancy (Figure 1a). Cohorts of $\operatorname{NHERF1}(+/+),(+/-)$ and $(-I-)$ 4-week-old nulliparous females were mated with $\operatorname{NHERF1}(+/+)$ males. Compared with $\operatorname{NHERF1}(+/+)$ and $(+/-)$ females that attained almost $100 \%$ delivery success rates by 11 weeks, only $73 \% \operatorname{NHERF1(-/-)}$ females delivered by 14 weeks. The remaining $\operatorname{NHERF1(-/-)}$ females eventually delivered by 20 weeks. The age at which $50 \%$ females delivered was delayed by 1 week for NHERF1 $(-/-)$ versus $(+/+)$ mice and was intermediate for heterozygous mice. These initial results indicated deficiencies in either fertilization, implantation or both in NHERF1 (- / - ) females.

A more severe cause for the breeding deficiency of $N H E R F 1(-/-)$ females appeared related to offspring survival after birth. Of note is that as only wild-type males were used for crosses, the resulting pups were either NHERF1 heterozygous or wild type. Wild-type females gave birth and kept litters of six or more pups with very few exceptions in which, at the first pregnancy, they ate their pups (Figure 1b). In contrast, approximately $50 \%$ of NHERF1 $(+/-)$ females failed to keep a full-size litter. Of these, twothirds of female mice lost the whole litter after birth and onethird were able to keep some pups (Figure 1b). The situation

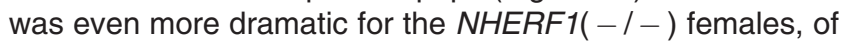
which $82 \%$ either completely or partially lost the litter (Figure 1b). The average litter size at birth was roughly equivalent among genotypes (Figure 1c). Pup mortality for NHERF1-mutant mothers was not subsequent to pup eating and was independent of pup genotype. Most newborn pups died within $24 \mathrm{~h}$ postpartum and appeared dehydrated but without other defects, whereas some pups failed to thrive and died few days later. As a rule, a significant number of surviving pups from NHERF1 $(-/-)$ mothers suffered growth retardation (Figure 1d) and were weaned 1-2 weeks later than those from $\operatorname{NHERF1}(+/+)$ mothers. These observations suggested a breast-feeding deficit in the failing mothers. This deficit appeared inversely correlated with the NHERF1 allele copy number, with failure rates of approximately 50 and $80 \%$ for $\operatorname{NHERF1}(+/-)$ and $\operatorname{NHERF1}(-/-)$ females, respectively.

NHERF1 expression is upregulated in the lactating mammary gland and required for its development. To characterize the mammary gland lactation deficit in NHERF1-mutant mice, we compared the morphology of mammary glands within a 24-h postpartum interval in mothers of the three genotypes that were either fully successful (termed lactation success) or fully unsuccessful (lactation failure) in nursing their litters. Whole-mount preparations of mammary glands showed full development of mature alveolar glands in $\operatorname{NHERF1}(+/+)$ and $(+/-)$ lactation success mothers, as well as in the rare successful $\operatorname{NHERF1}(-/-)$ cases (Figure 2a). In contrast, the glands from NHERF1-mutant mothers with lactation failure did not develop mature alveoli characteristic of the late pregnancy/ lactating gland. As control, mammary glands from agematched virgin females showed grossly comparable development of the ductal system for all three genotypes. The developmental arrest was confirmed also by histopathology that showed the presence of numerous and large milkproducing alveoli in lactation-success glands, and of fewer and less expanded alveoli in lactation-failure glands (Figure $2 \mathrm{~b}$ and Supplementary Figure S1). Importantly, the glands with lactation failure did not show atrophy, dilatation or sloughing of apoptotic cells in the lumen, features that characterize the postlactational regression due to cessation of breastfeeding. The absence of apoptosis was confirmed by performing in situ apoptosis detection (not shown), indicating that the changes observed in the lactation-failure glands were consistent with a maturation deficit. These findings indicated that NHERF1 is required for the expansion and full maturation of the alveoli in the mammary gland during pregnancy.

A key experiment consisted in assessing NHERF1 expression in virgin, lactation-success or lactation-failure mammary glands (Figures 2c and d). Strikingly, NHERF1 protein levels were massively upregulated in $\operatorname{NHERF1}(+/+)$ or $(+/-)$ lactation-success glands and depressed in $\operatorname{NHERF1}(+/-)$ lactation-failure glands (Figure 2c). This strongly supported an essential role for NHERF1 in lactation. As expected, NHERF1 $(-/-)$ glands showed no NHERF1 protein or mRNA (Figures $2 \mathrm{c}$ and $\mathrm{d}$ ). We then traced the lactationdependent variations of NHERF1 protein expression to variations of mRNA expression by RT-PCR or quantitative PCR (Figure 2d). Interestingly, NHERF1 mRNA levels correlated with both allele copy number and gland differentiation status, being the highest in the wild-type lactating glands. Consistent with the lactation impairment in NHERF1-mutant animals, NHERF1 mRNA levels in NHERF1 + + / - ) lactationfailure glands were significantly lower than in lactationsuccess glands, suggesting perhaps a positive feedback loop in the transcription of NHERF1, dependent on the differentiation status of the mammary gland.

To explain the rare cases of lactation success in $\operatorname{NHERF1}(-/-)$ dams, we hypothesized that NHERF2, the homolog of NHERF1, ${ }^{23}$ may compensate for NHERF1 loss. 

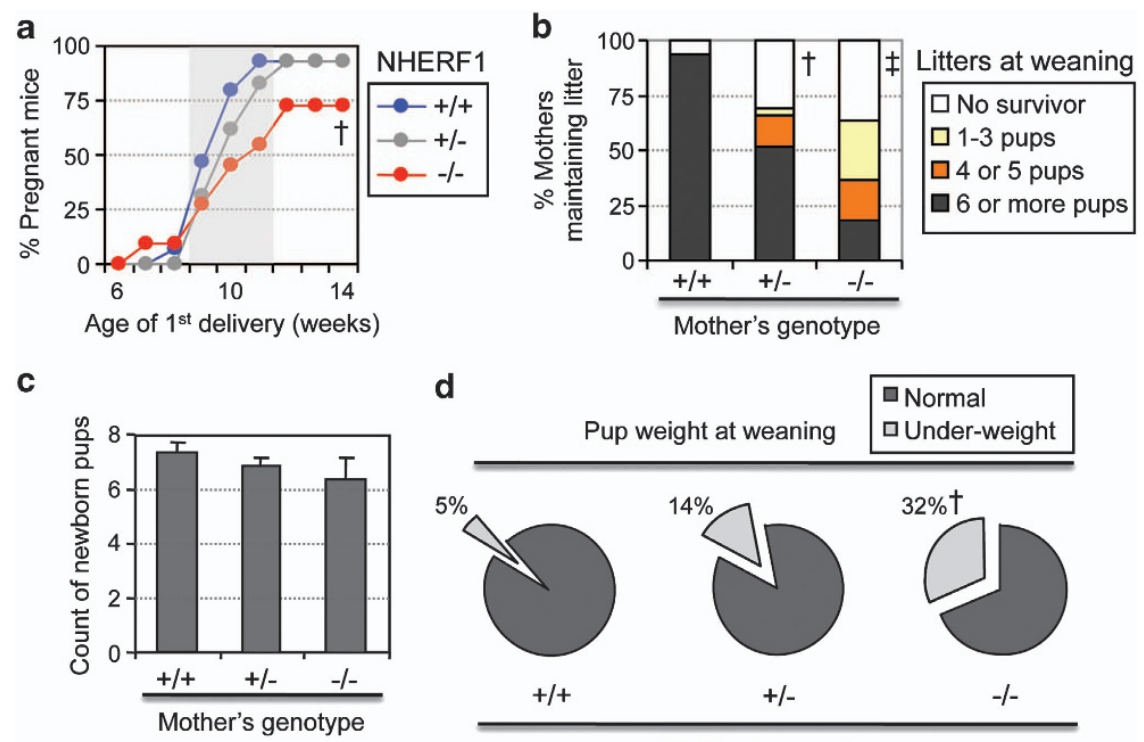

d

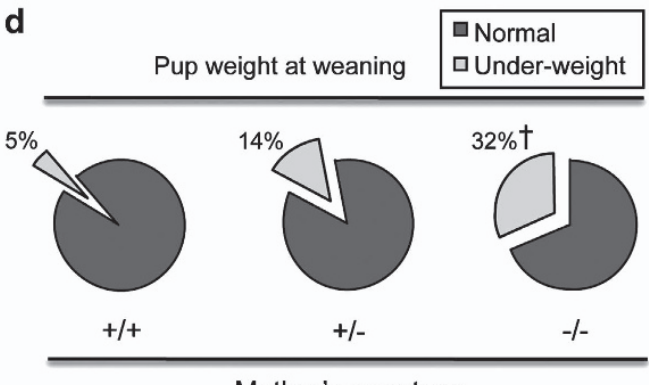

Mother's genotype

Figure 1 Impaired reproductive performance of NHERF1-mutant female mice. (a) Cumulative frequency of first pregnancy in $N H E R F 1(+/+)(n=15),(+/-)(n=29)$ and $(-I-)(n=11)$ females. Gray-shaded area indicates peak ages of frequency accumulation. (b) The ability of the dams from a to nurse their litter is represented as the distribution litter sizes per mother's genotype. (c) Newborn count per mother's genotype. (d) Pup weight per mother's genotype. The lower limit for the normal weight was arbitrarily defined as (mean - 1.5 S.D.) of weaning body weights from five NHERF1( $+/+)$ litters (day $21, n=42$ pups). For $(\mathbf{a}-\mathrm{d}),{ }^{\dagger} P<0.05,{ }^{\ddagger} P<0.01$, versus $(+/+$ )

We found that the NHERF2 levels were raised in the NHERF1 $(+/+)$ and $(+/-)$ lactation-success glands, similarly to NHERF1, whereas they were not upregulated in lactation-failure glands (Figure 2e). Interestingly, in the NHERF1(-/ - ) lactation-success glands, NHERF2 levels were elevated, suggesting that in these rare instances, NHERF2 might compensate for NHERF1 loss.

Akt activation correlates with NHERF1 genotype in nonlactating glands. As we have previously shown that

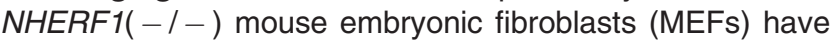
activated the PI3K-Akt pathway ${ }^{24}$ and proliferate at a higher rate than their wild-type counterparts, ${ }^{25}$ we examined the Akt activation in mammary glands (Figure 3). A first observation was that Akt activation is increased in the non-gestating mammary glands in an NHERF1 allele copy-dependent manner (Figure 3a), confirming a previous report in NHERF1-mutant virgin mice. ${ }^{26}$ The proliferation, as measured by 5-bromo-2'-deoxyuridine (BrdU) incorporation (Figure 3b), was significantly increased in $\operatorname{NHERF1(-/-)}$ versus $(+/+)$ ducts, suggesting direct correlation with the Akt activation in NHERF1-mutant non-gestational mammary glands.

In the lactating alveolo-lobular gland, we did not find increased Akt activation compared with the ductal nongestational gland, regardless of the mice genotype (Figure 3a). This finding eliminated the PI3K-Akt pathway as being responsible for the lactation deficit of NHERF1-mutant mice. However, in the lactating glands, there was a massive increase in proliferation compared with non-gestational glands, as detected by PCNA expression (Supplementary Figure S2), which was correspondingly reduced in lactationfailure glands. This pointed to the PRLR-STAT5 pathway as the developmental program involving NHERF1, as it triggers both proliferative and differentiation signals in the mammary gland during gestation.

PRLR interacts with NHERF1 and is depleted from the basal membrane of alveolar cells lacking NHERF1. PRLR is upregulated during gestation in the developing mammary gland. ${ }^{27}$ We observed a PRLR expression upregulation in lactation-success mammary glands and lack of this upregulation in lactation-failure glands (Figure 4a). The PRLR expression pattern mirrored NHERF1 expression, except for the rare cases of lactation success in NHERF1 (- / - ) glands, suggesting the possibility of a link between PRLR and NHERF1. NHERF1 is a PDZ-domain adapter protein that stabilizes its binding partners at the plasma membrane. ${ }^{18,28}$ We therefore examined PRLR for the presence of a PDZ motif and found a putative C-terminal candidate sequence conserved in human, rodent and swine proteins (Supplementary Table S1). This motif appeared to mediate the direct binding between recombinant NHERF1PDZ domains and PRLR $\mathrm{C}$ terminus, as its deletion abrogated the interaction (Figure 4b). By pull-down with glutathione S-transferase (GST)-PRLR of NHERF1-domain mutants, we found that all but the NHERF1-PDZ1-M2 mutant interacted with PRLR, thus identifying NHERF1PDZ1 domain as the main interaction module with PRLR (Figures $4 c$ and $d$ ).

PRLR was expressed at the basal plasma membrane in alveolar cells (Figure 4e). We found that NHERF1 colocalized with PRLR at the basal membrane of alveolar cells in lactation-success glands (Figure 4e). In lactation-failure glands from matched $N H E R F 1(+/-)$ females, the basal membrane localization of both proteins was virtually abolished 
a

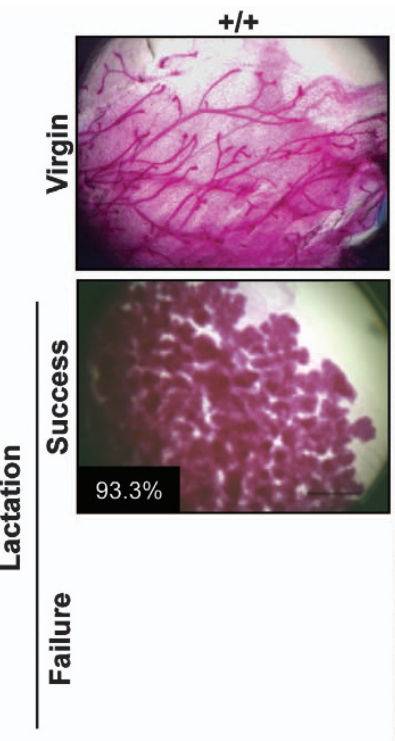

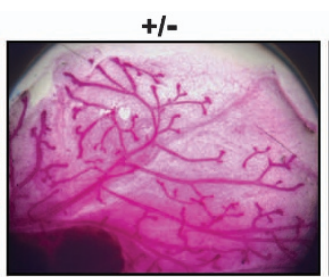
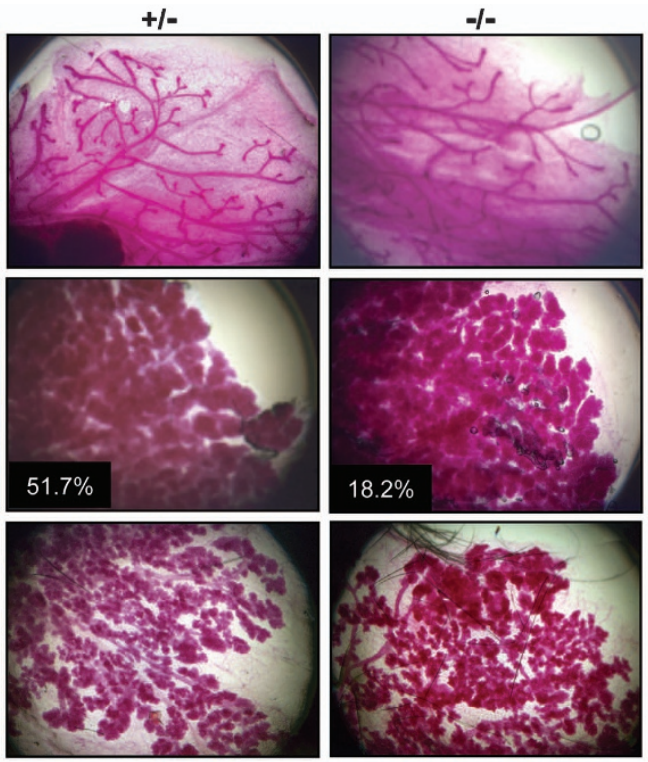

C
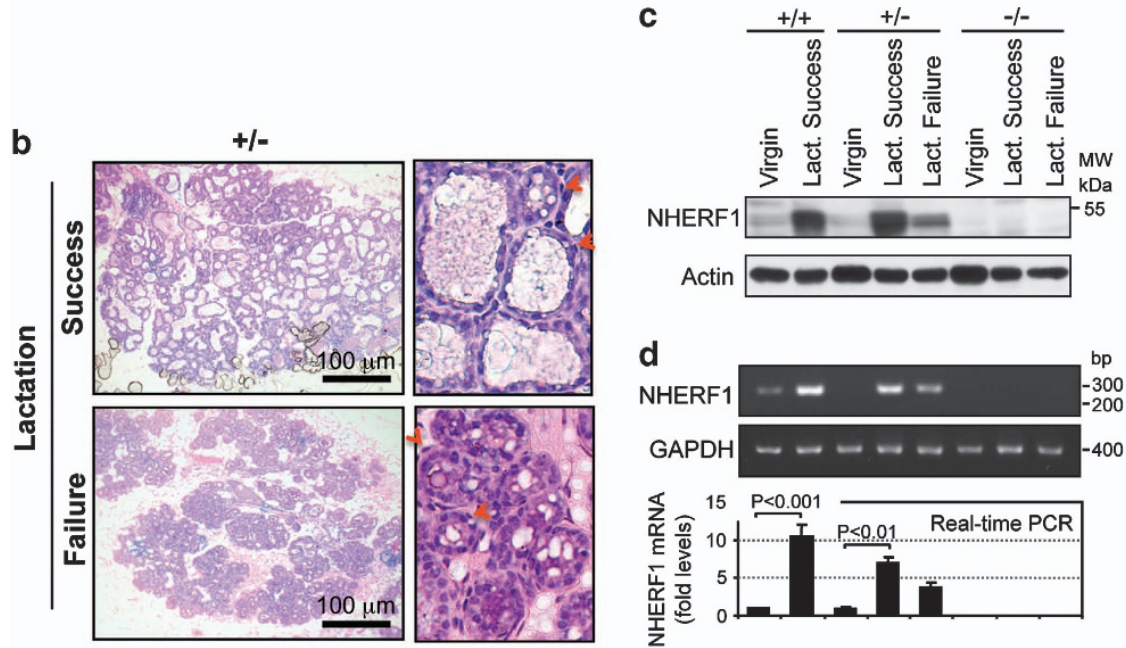

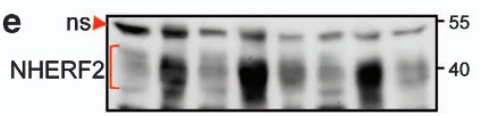

Figure 2 Impaired mammary gland development correlates with lack of NHERF1 upregulation during pregnancy. (a) Whole-mount analysis by carmine alum staining of mammary glands from age-matched NHERF1 $(+I+),(+I-)$ and $(-I-)$ virgin females and dams at $\leq 24 \mathrm{~h}$ postpartum. Boxed numbers indicate the success rates for nursing six or more pups (from Figure 1b). (b) Hematoxylin and eosin staining of contralateral mammary glands from lactation success and lactation failure mothers at $\times 100$ and $\times 200$ magnification. Myoepithelial cells are shown with arrowheads. (c) Western blot of mammary gland lysates shows NHERF1 upregulation in lactation success glands. Proteins were loaded at $100 \mu$ g per lane. (d) NHERF1 mRNA analysis by RT-PCR (panels) or quantitative real-time PCR (graph). $n=3$. (e) Western blot with NHERF2 antibody shows compensatory NHERF2 upregulation in NHERF1 $+(-)$ and $(-/-)$ success glands. NS, nonspecific band

(Figure 4e, lower panels), indicating that NHERF1 is required for the stabilization of PRLR at the basal membrane. Of note is that in ducts from adult virgin females, NHERF1 has apical localization in luminal cells, as previously reported ${ }^{21,22}$ (Supplementary Figure S3).

NHERF1 assembles macromolecular complexes with PRLR, $\beta$-catenin, E-cadherin and ezrin, and maintains alveolar basal membrane polarity. As the morphology of the alveoli is altered in NHERF1-mutant glands (Figure 2b), we examined whether NHERF1 loss could have a more generalized effect on protein complex formation and membrane polarity than just the displacement of PRLR alone. NHERF1 binds to $\beta$-catenin through the PDZ2 domain ${ }^{29}$ (Figure 4d), and we have previously shown that the plasma membrane localization of $\beta$-catenin-E-cadherin complexes is disrupted in NHERF1 $(-/-)$ MEFs. ${ }^{25}$ To examine these complexes in mammary alveolar cells, we co-immunoprecipitated PRLR from cell lysates of lactation-success glands and observed that PRLR, NHERF1, $\beta$-catenin and E-cadherin associate in complex (Figure $5 \mathrm{a}$ ). We confirmed this finding by co-immunoprecipitating the same complex from 

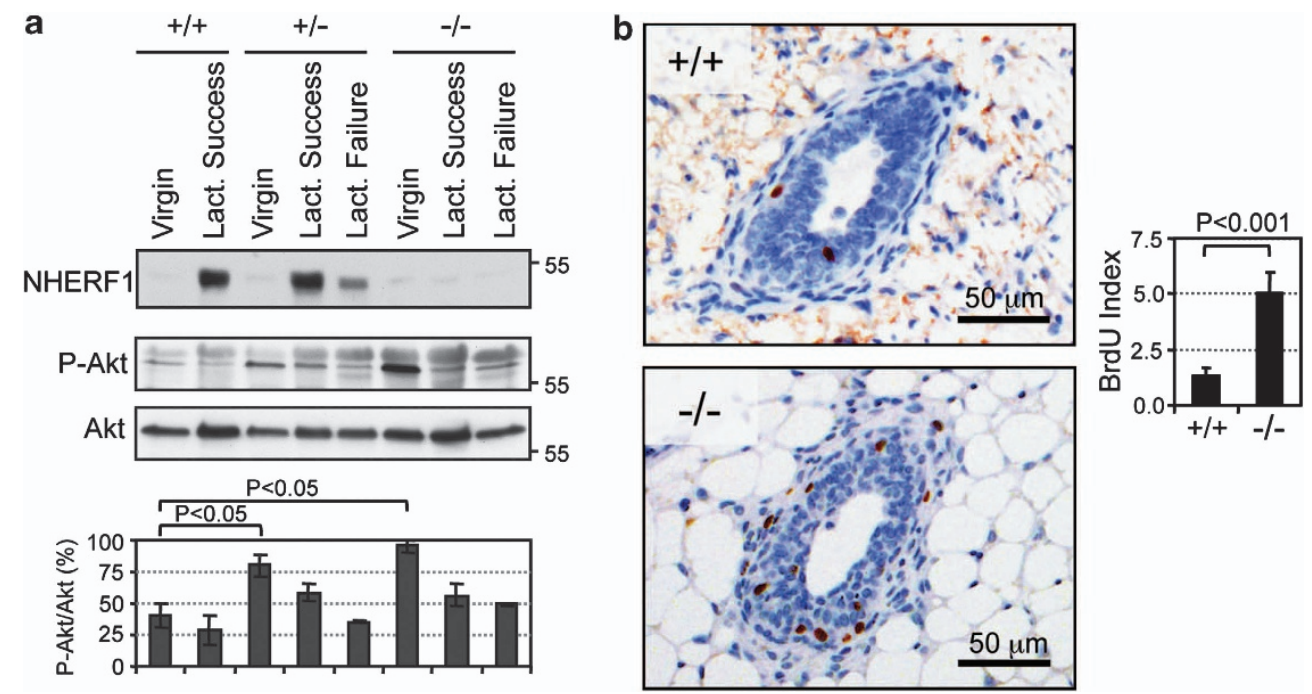

Figure 3 NHERF1 loss increases Akt activation and proliferation in mammary ducts. (a) Western blot of mammary gland lysates as in Figure $2 c$. The graphs show the densitometric intensities of phosphorylated S473 Akt levels normalized to total Akt levels. $n=2$ blots. (b) BrdU staining of mammary gland in 6-week-old NHERF1( + I +) and $(-/-)$ virgin littermates. The graph shows the normalized BrdU-positive cell count per 0.2-mm² mammary duct area. $n=35$ ducts

human T47D breast cancer cells that express endogenous PRLR (Figure 5b). Furthermore, we analyzed the patterns of expression of these proteins in lactation-success or lactationfailure glands and observed that they mirror the PRLR/ NHERF1 expression pattern (Figure $5 \mathrm{c}$ ). In addition to $\beta$ catenin and E-cadherin, ezrin that binds to NHERF1 ERMbinding region $^{15}$ (Figure $4 d$ ) also had similar expression pattern (Figure 5c) and co-immunoprecipitated with NHERF1 in mammary alveolar cells (not shown). These findings suggested that NHERF1 assembles larger complexes at the basal membrane of alveolar cells that involve PRLR and structural or cytoskeletal proteins.

To estimate the degree of disruption of the alveolar basal membrane, we analyzed the distribution of E-cadherin, as a marker of basolateral cell polarity, and of laminin, as marker for basement membrane integrity (Figure 5d). Both E-cadherin and laminin were significantly reduced from the basal aspect of the alveoli in $N H E R F 1(+/-)$ lactation-failure glands versus $N H E R F 1(+/-)$ lactation-success glands. Interestingly, the lateral membrane distribution of E-cadherin was preserved in the absence of NHERF1 (Figure $5 \mathrm{~d}$, graph), and in some alveoli, an inversion of the basal to apical membrane distribution was observed (Figure $5 \mathrm{~d}$, right panels). Taken together, these data indicate a severe disruption of basal membrane polarity by NHERF1 loss.

STAT5 and Elf5 signaling are altered in undifferentiated NHERF1-mutant alveoli. We further investigated whether the impairment of PRLR expression and localization in NHERF1-mutant glands impacts on STAT5 activation. STAT5 was clearly phosphorylated only in lactation-success glands (Figures 6a and d). Strikingly, phosphorylated STAT5 was present in both the cytoplasm and nuclei of lactating alveolar cells, suggesting intense transcriptional activity, whereas it was essentially absent from the alveoli of lactation-failure glands (Figure $6 \mathrm{~d}$ ). The expression of cyclin D1, an important STAT5 transcriptional target, paralleled closely STAT5 phosphorylation pattern, consistent with the differential activation of STAT5 (Figure 6b). In addition, the transcription factor Elf5, another crucial transcription factor for lobuloalveolar development downstream of PRLR signaling, ${ }^{30}$ was also specifically upregulated in lactation-success glands (Figure 6c). In cell lines, Erk has also been shown as downstream mediator of prolactin signaling. ${ }^{31}$ However, Erk had a different activation pattern, being activated during pregnancy in both lactation-success and lactation-failure glands (Supplementary Figure S4), and thus implicating the PRLR-STAT5/Elf5 as the main pathway controlled by NHERF1 for the development of the functional gland during pregnancy.

The ultimate markers of differentiation of a functional secreting mammary gland are the milk proteins, which are also direct STAT5 transcriptional targets. As expected, the expression of $\beta$-casein, whey acidic protein (WAP) and $\alpha$-lactalbumin followed the pattern of STAT5 activation (Figure 6e). Notably, the apparent lesser activation of STAT5 in the rare cases of $N H E R F 1(-/-)$ lactation-success glands (Figure 6a) was translated in consistently lesser transcription levels of the milk proteins (Figure 6e). This finding indicates incomplete differentiation of $\operatorname{NHERF1}(-/-)$ lactationsuccess glands that most likely resulted in poor milk quality and failure to thrive of $32 \%$ of surviving pups from lactating $\operatorname{NHERF1}(-/-)$ dams (Figure 1d).

\section{Discussion}

The lactating function of the mammary gland in mammals is absolutely essential for newborn survival. To execute it, a complex developmental process takes place during pregnancy, leading to the differentiated secreting lobulo-alveolar mammary gland. The many signals necessary for its completion seemingly converge onto a central pathway orchestrated around the PRLR-STAT5 axis. The molecules that modulate or are part or this pathway are required for the proliferation and 

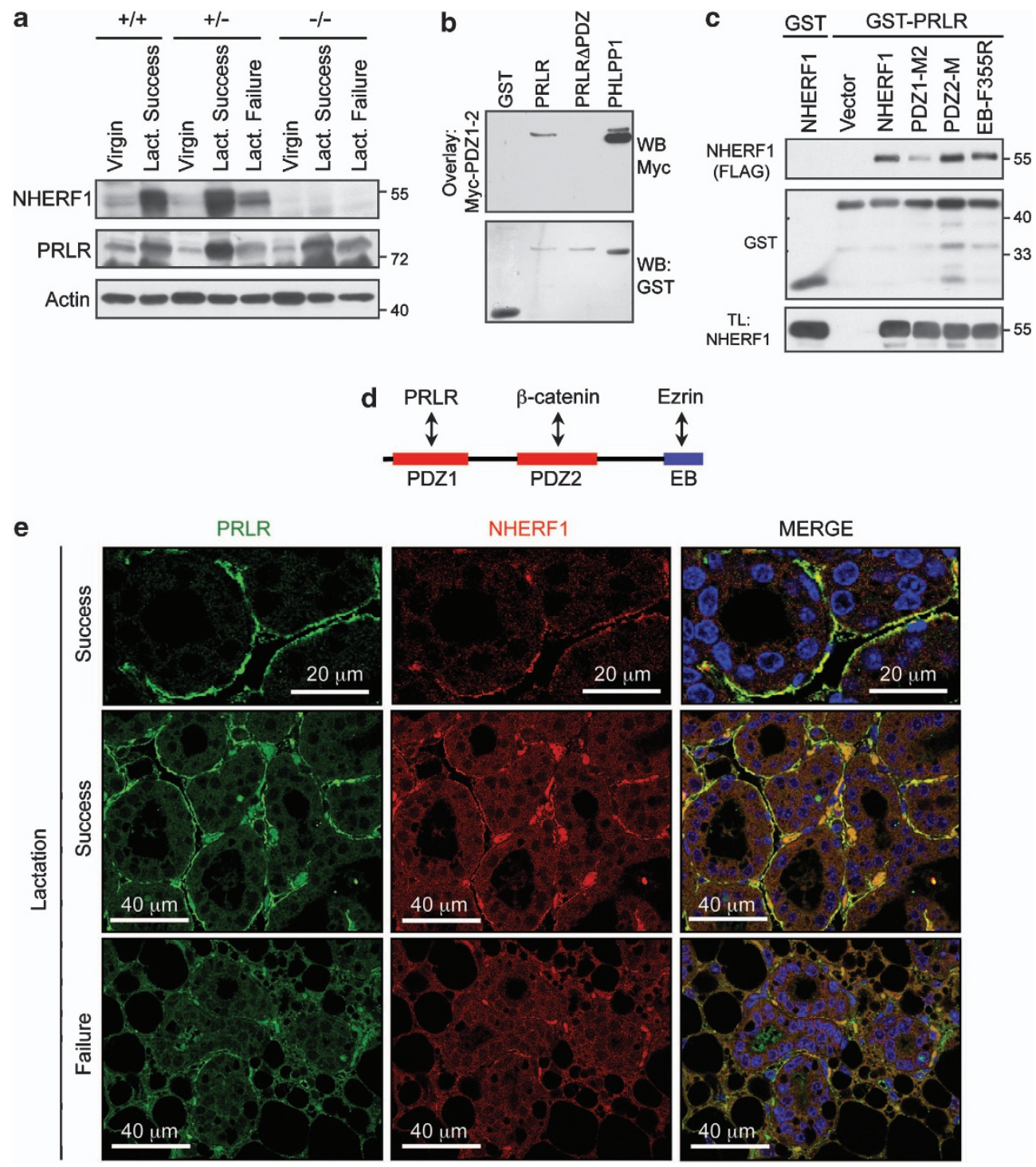

Figure 4 NHERF1 interacts with PRLR and is required for PRLR localization at the basal membrane of alveolar cells. (a) Western blot of mammary gland lysates with indicated antibodies. (b) Overlay assay showing direct interaction between filter-immobilized GST-PRLR C terminus with intact PDZ motif (PRLR) and overlaid Myc-tagged NHERF1-PDZ1-2 domains. The filter was probed with Myc antibody to detect the interaction, stripped and re-probed with GST antibody, to show the amount of GST fusion proteins loaded. PRLR $\triangle P D Z$, GST-PRLR C terminus mutant with PDZ-motif deletion. GST and GST-PHLPP1 are negative and positive interaction controls with NHERF1PDZ1-2, respectively. ${ }^{43}$ (c) GST pull-down with GST control and GST-PRLR proteins $(4 \mu \mathrm{g})$ on total lysates $(0.7 \mathrm{mg})$ of $293 \mathrm{~T}$ cells transfected with vector and the indicated FLAG-tagged NHERF1 constructs. TL, input total lysate $(25 \mu \mathrm{g})$. (d) Schematic organization of NHERF1 PDZ domains and ERM-binding (EB) region, and their corresponding interactions discussed in this study. (e) Confocal IF analysis with PRLR (green) and NHERF1 (red) antibodies of lactation success and lactation failure NHERF1 ( $/$ - ) mammary glands. Nuclei were stained with ToPro-3 (blue). Note co-localization of NHERF1 and PRLR at the alveolar basal membrane in lactation success glands

terminal differentiation of the alveoli, resulting in the production of milk after delivery. We show here that NHERF1 is a new component of the central PRLR-STAT5 pathway and functions by locating PRLR at the basal plasma membrane of alveolar cells where it can be bound and activated by prolactin (Figure 7).

NHERF1 has been previously involved in stabilizing transmembrane proteins either by inhibiting their endocytosis or by enhancing their recycling to the membrane. ${ }^{28,32,33}$ Although either of these mechanisms could operate for PRLR, it appears that NHERF1 has a broader role in mammary alveoli in which it coordinates the establishment of basal membrane polarity. The maintenance of cell polarity has been shown to be an important factor for the differentiation of alveolar cells as mammary gland-targeted deletion of E-cadherin or connexin-26, an essential integral component of gap junctions, prevented the development of functional glands during pregnancy. ${ }^{12,34}$ At the basal membrane, NHERF1 binds via its PDZ1 domain to PRLR, and together are part of macromolecular complexes comprising the cell-adhesion E-cadherin- $\beta$-catenin assemblies and the cytoskeletal protein ezrin. NHERF1 associates to $\beta$-catenin via its PDZ2 domain ${ }^{29}$ and to ezrin via its ERM-binding region, ${ }^{15}$ suggesting the possibility of heteromeric complexes with PRLR. We have 
a

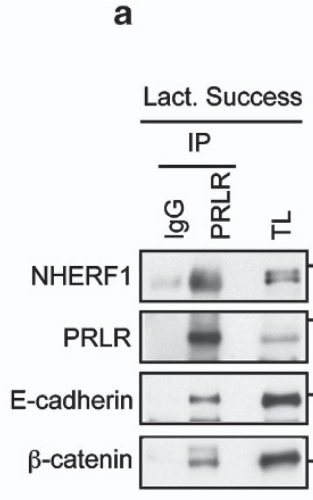

b

c
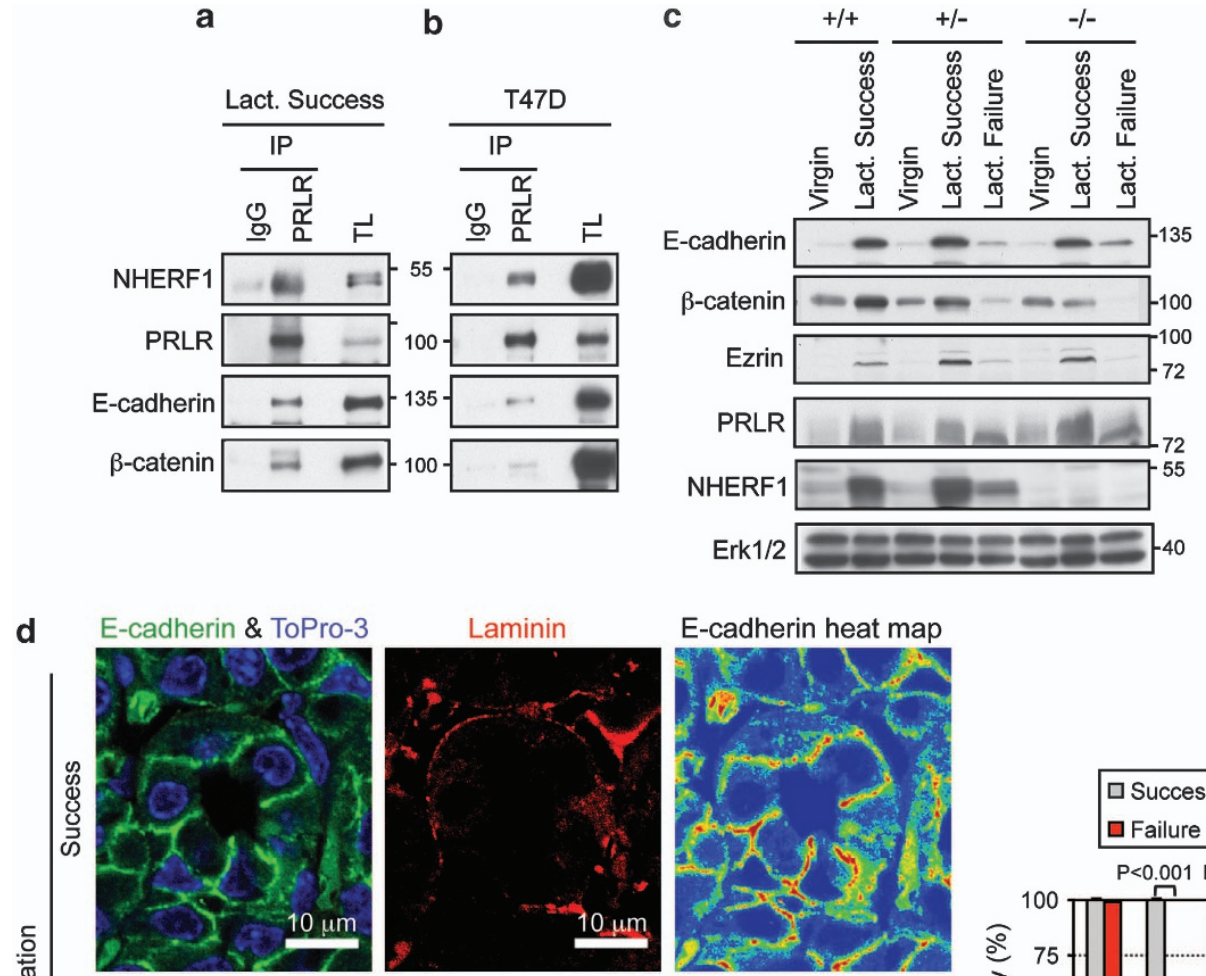

E-cadherin heat map
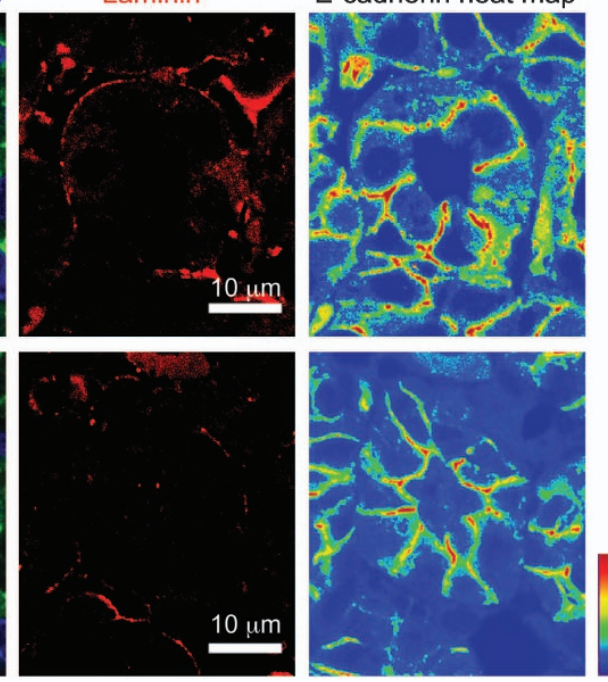

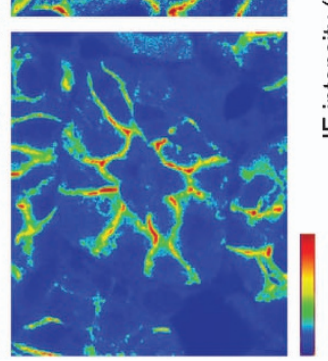

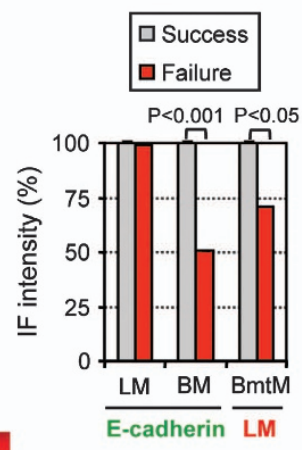

Figure 5 NHERF1 assembles macromolecular complexes at the basal membrane of alveolar cells. (a and b) Immunoprecipitation (IP) of NHERF1, E-cadherin and $\beta$ catenin with PRLR or control lgG antibodies from total lysates (TL) of lactation success mammary glands (a) or T47D breast carcinoma cells (b). (c) Western blot of mammary gland lysates with indicated antibodies. Erk1/2 levels are shown as loading control. (d) Confocal IF analysis with antibodies for E-cadherin (green), laminin (red) and with ToPro-3 (blue) of lactation success and lactation failure NHERF1 + I - ) mammary glands. The E-cadherin heat scale shows the baso-apical shift of E-cadherin staining intensity in lactation failure alveoli. The graph shows the comparisons of E-cadherin and laminin (LM) IF densitometric intensities. $n=30$ cells. LM, lateral membrane; BM, basal membrane, BmtM, basement membrane

previously shown that such complexes involving distinct PDZ domain ligands and ezrin can be formed and that the binding of ezrin enhances the affinity of NHERF1 for PDZ-domain ligands. ${ }^{35}$ Interestingly, these macromolecular complexes could couple the actin cytoskeleton either through ezrin via its actin-binding tail $^{36}$ or through a $\beta$-catenin- $\alpha$-catenin interaction, ${ }^{37}$ thus conferring the resistance structure for the basal membrane (Figure 7). An extra layer of compartmentalization could be added by the special partition of the basal plasma membrane in lipid rafts. NHERF1 has been shown to reside in lipid rafts ${ }^{38}$ and caveolin-1, a structural component of lipid rafts, and has been involved in the termination of the PRLR signaling, probably by sequestering JAK2 ${ }^{39}$ It is thus tempting to speculate that NHERF1 could antagonize the effects of caveolin-1 by segregating PRLR in distinct macromolecular complexes in lipid rafts.

The expression of NHERF1 is closely regulated in alveolar cells during pregnancy and rises in parallel to the PRLR expression but also to that of the other structural components of the macromolecular complex. This whole program could be regulated through the STAT5 network, ${ }^{1}$ as we detected conserved STAT5 transcription sites in NHERF1 human and mouse promoters (not shown). Interestingly, in the rare NHERF1 ( - / - ) lactation-success cases, where no NHERF1 protein is present in cells, all the components of the macromolecular complex are upregulated and probably forming a complex. In these cases, STAT5 signaling and milk protein synthesis are present albeit reduced, suggesting compensation for NHERF1 loss, even if not complete. This compensation appears to be random, as initially successful NHERF1 ( - / - ) dams failed in next pregnancies. NHERF2, which interacts with both ERM proteins and $\beta$-catenin, ${ }^{35}$ is upregulated in $N H E R F 1(-/-)$ lactation-success glands, supporting a compensatory role in these cases.

From our study, the signaling through PRLR-STAT5 appeared to be the main force driving the differentiation but 
a

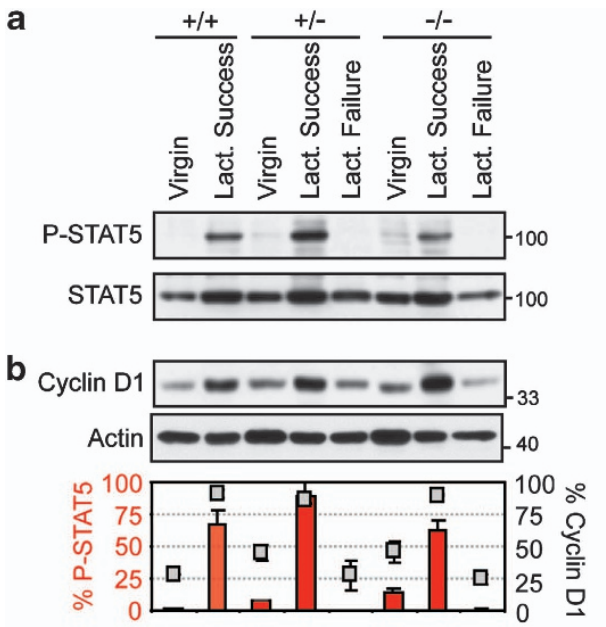

c
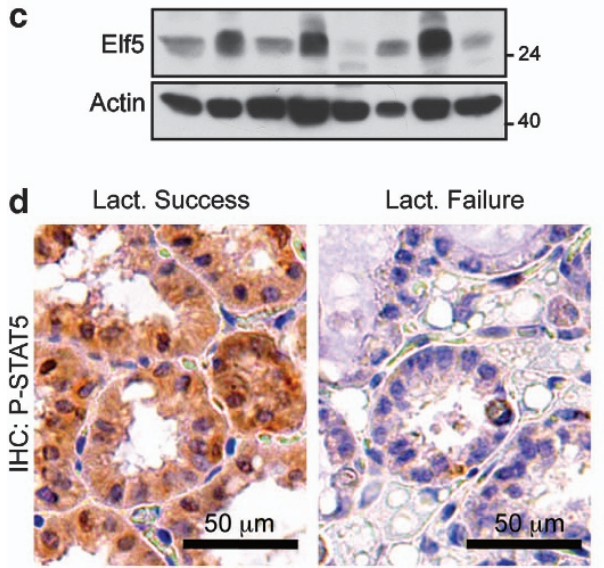

e
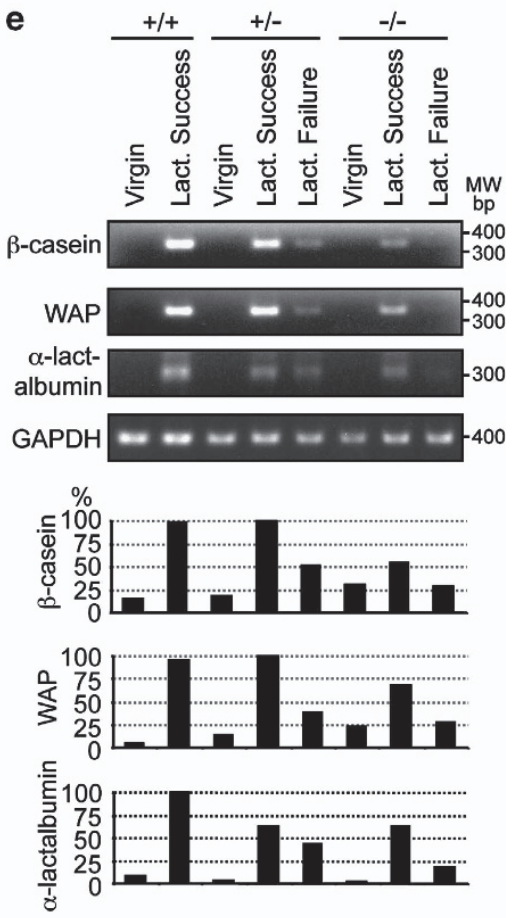

Figure 6 STAT5 activation is impaired in the lactating mammary gland of NHERF1-mutant mice. (a-c) Western blot of mammary gland lysates with indicated antibodies. The graph shows the densitometric intensities of normalized phosphorylated P-STAT5/total STAT5 and Cyclin D1/actin levels. $n=2$ blots. (d) Immunohistochemical analysis of NHERF1 $(+/-)$ mammary glands showing P-STAT5 in the cytoplasm and nucleus of lactation success glands. (e) Milk proteins mRNA analysis by RT-PCR. The graph shows the densitometric intensities of GAPDH-normalized $\beta$-casein, WAP and $\alpha$-lactalbumin levels

also the proliferation of the lactating mammary gland, as proliferation markers, such as PCNA and cyclin D1, $5,10,11$ were upregulated only in the mammary glands with lactation success and activated STAT5. Intriguingly, the mitogenic Ras-Erk pathway that was also reported to be downstream of $\mathrm{PRLR}^{31}$ was also activated in lactation-failure glands, implying a different upstream trigger than PRLR in these cases. Perhaps, the signals through Erk are required but certainly not sufficient for the development of the lactating mammary gland. Surprisingly, the PI3K-Akt pathway that was previously shown to be suppressed by NHERF1 through recruitment of PTEN tumor suppressor, ${ }^{24}$ did not appear to be upregulated in the mammary gland during pregnancy. However, in mature virgin glands, Akt activation and the proliferation of duct cells were clearly correlated with NHERF1 allele copy number, supporting a mechanistic explanation for the involvement of NHERF1 in breast cancer. Indeed, NHERF1 loss-of-heterozygosity has been documented in breast cancer cell lines and samples, ${ }^{40}$ and the related PI3K-Akt pathway activation has been implicated in breast cancer pathogenesis. ${ }^{26}$

In contrast to a gradual Akt activation increase dependent on a haploinsufficiency effect of NHERF1 dosage in the predominantly ductal gland of non-pregnant females, the development of the gestational lobulo-alveolar gland appeared to be an all-or-none phenomenon. For example, most of NHERF1 $(+/-)$ dams either had fully differentiated mammary glands and could keep full litters or had developmental failure with whole litter loss. The NHERF1 protein levels are consistent with this mechanism, being as elevated in lactation-success $\operatorname{NHERF1}(+/-)$ glands as in their $N H E R F 1(+/+)$ counterparts. On the other hand, the NHERF1 mRNA levels are dependent on both NHERF1 genotype and gland phenotype, suggesting the need for a certain mRNA threshold for mammary gland development. Upon reaching this threshold, posttranslational mechanisms or rather cellular/membrane compartmentalization appear to stabilize NHERF1 to wild-type protein levels. If this scenario is

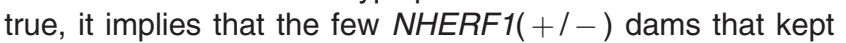
partial litters had a mixture of developed and undeveloped mammary glands, and we are currently investigating this possibility. This explanation is in agreement with the development of the lactating mammary gland from alveolar precursor cells ${ }^{41}$ that may have different levels of NHERF1 mRNA transcripts in different glands. 


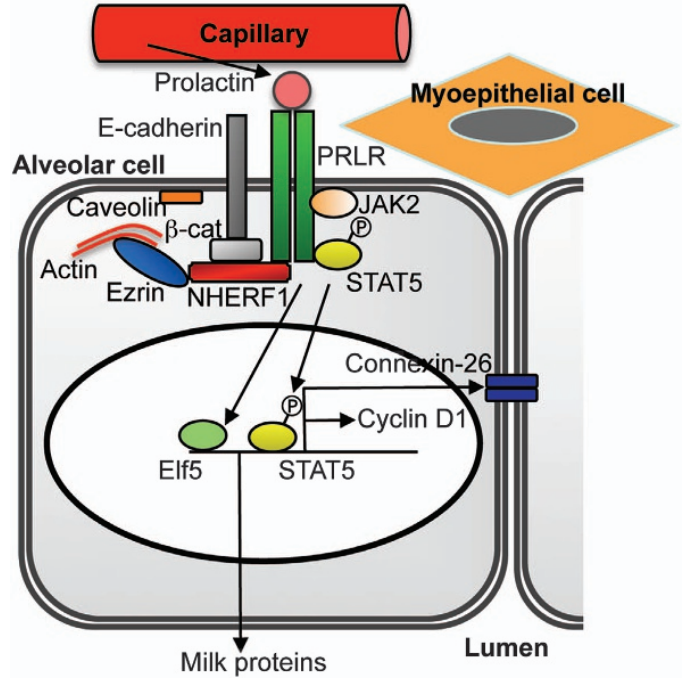

Figure 7 Model depicting the role of NHERF1 in the lactating mammary gland. For simplicity, the symmetry/stoichiometry of the PRLR-JAK2-STAT5 complexes is not shown

In conclusion, we found that NHERF1, an adapter protein previously implied in the progression of breast cancer, either by loss or cytoplasmic mislocalization, ${ }^{18,20-22,40}$ has a physiological and essential function in the development of the lactating mammary gland. Through expression upregulation and strategic localization at the basal membrane of alveolar cells, NHERF1 engages PRLR in structural complexes that maintain the polarity and directional signaling via STAT5 activation. Further studies aimed at the regulation of NHERF1 gene transcription, and protein stability could shed light on the pathogenesis of breast cancer, but also lead to improvements in the secretion and quality of milk.

\section{Materials and Methods}

Mice and whole-mount staining of mammary glands. The NHERF1-deficient mice ${ }^{14}$ used for these studies were inbred for 10 generations in C57BL/6J background. Pregnant NHERF1 $(+/+),(+/-)$ and $(-/-)$ agematched females were followed up daily for delivery. The litters were examined within $24 \mathrm{~h}$ postpartum, and the number of dead and surviving pups was recorded. The mammary glands were dissected only from females that either kept or lost the entire litter within the first $24 \mathrm{~h}$ postpartum. The consensus numbering of the five pairs of mammary glands and the procedures applied are shown in Supplementary Figure S5. The left inguinal mammary glands were removed, spread onto glass slides and fixed overnight in Carnoy's fixative $(75 \%$ ethanol, $25 \%$ glacial acetic acid). The samples were washed in $70 \%$ ethanol for $15 \mathrm{~min}$, hydrated in double distilled water and stained overnight in carmine alum solution ( $2 \%$ carmine (w/v), $5 \%$ aluminum potassium sulfate $(\mathrm{w} / \mathrm{v}))$. The samples were further dehydrated in 70, 95 and $100 \%$ ethanol for 15 min each, then cleared in xylene.

BrdU labeling. NHERF1 $(+/+)$ and $(-/-)$ mice were injected intraperitoneally with $0.1 \mathrm{ml} / 10 \mathrm{~g}$ body weight of BrdU (Invitrogen, Carlsbad, CA, USA) and killed $2 \mathrm{~h}$ later. The left and right inguinal mammary glands were removed and spread onto glass slides. After overnight fixation in 10\% formalin and paraffinembedding, 4- $\mu \mathrm{m}$ sections were obtained for staining with the Animal Research Kit (Dako, Carpinteria, CA, USA).

Histology and immunostaining. The right inguinal mammary glands were removed, spread onto glass slides and fixed overnight in 10\% formalin. After embedding in paraffin, 4- $\mu \mathrm{m}$ serial sections were processed by hematoxylin and eosin, immunohistochemistry or immunofluorescence (IF), as previously described. ${ }^{42,43}$ The primary antibodies were: NHERF1 (Abcam, Cambridge, MA, USA) at 1:300, PRLR (Affinity BioReagents/Thermo, Rockford, IL, USA) at 1:50, E-cadherin (BD Biosciences, LaJolla, CA, USA) at 1:50, laminin (Sigma-Aldrich, St Louis, MO) at 1:50 and phospho-STAT5-Y694 (no. 9134, Cell Signaling Technology, Danvers, MA, USA) at 1:50. The densitometric analysis of the IF intensity was performed with the ImageJ software version $1.34 \mathrm{~s}$ (National Institutes of Health, Bethesda, MD, USA).

RNA analysis. Total RNA was extracted from the left thoracic mammary gland with a pestle in TRIzol Reagent (Invitrogen). Reverse transcription was performed with $750 \mathrm{ng}$ RNA to generate first-strand cDNA using the iScript cDNA Synthesis kit (Bio-Rad, Hercules, CA, USA). One-tenth of the CDNA was used as a template for PCR amplification. The primer sequences for NHERF1, $\beta$-casein, WAP, $\alpha$ lactalbumin and GAPDH are provided in Supplementary Material. For NHERF1 and GAPDH, real-time PCR was performed using IQ SYBR Green Supermix in the Chromo4 system (Bio-Rad). Group differences in GAPDH-normalized NHERF1 mRNA levels were estimated using the $2^{\wedge}(\Delta \Delta \mathrm{Ct})$ formula.

Cells, plasmids and transfection. The human T47D breast carcinoma and 293 T cells were grown in DMEM/F-12 supplemented with $10 \%$ FBS. The human PRLR full-length cDNA was purchased from Open Biosystems/Thermo (clone 30343995). The C-terminal 173 residues of PRLR cytoplasmic domain were inserted in the pGEX-6P1 vector in frame with GST for recombinant protein purification from bacteria. The deletion mutant lacking the last three residues that constitute the PDZ motif, PRLR $\triangle$ PDZ, was obtained by PCR. Human NHERF1 wild-type and ERM-binding region mutants $\triangle$ SNL and F355R in pFLAG-CMV-2 vector have been described previously. ${ }^{35}$ The PDZ-domain point mutants NHERF1-PDZ1M2 and NHERF1-PDZ2M, which have disrupted the PDZ-domain binding pockets by GF to AA substitutions at positions $25-26$ and 165-166, respectively, were obtained by PCR. The constructs were transiently transfected in $293 \mathrm{~T}$ cells as previously described. ${ }^{24}$

Protein analysis. Proteins were extracted from the right thoracic mammary gland in lysis buffer ( $2 \%$ Triton X-100, $50 \mathrm{mM}$ HEPES, pH 7.5, $100 \mathrm{mM} \mathrm{NaCl}$, $10 \mathrm{mM}$ EDTA and $10 \%$ glycerol) with a pestle. After clarification at $16100 \times \mathrm{g}$ for $15 \mathrm{~min}$ at $4{ }^{\circ} \mathrm{C}$, the protein concentration was measured using the BCA Protein Assay kit (Thermo). For immunoprecipitation, $500 \mu \mathrm{l}$ lysates containing $4 \mathrm{mg}$ proteins were incubated overnight at $4{ }^{\circ} \mathrm{C}$ with either $4 \mu \mathrm{g}$ PRLR antibody or control rabbit IgG. Protein-G Dynabeads ( $100 \mu \mathrm{l}$; Invitrogen) were added for $45 \mathrm{~min}$. Immune complexes were collected by centrifugation at $4800 \times \mathrm{g}$ for $2 \mathrm{~min}$, and washed three times in lysis buffer. The immunoprecipitation of endogenous proteins from T47D cells was performed similarly by using $1 / 4$ of the protein amount and reagents. Western blot, overlay and GST pull-down assays were performed as described. ${ }^{24}$ The primary antibodies were as follows: NHERF1 (Calbiochem/Millipore, Billerica, MA, USA); E-cadherin, $\beta$-catenin (BD Biosciences); phospho-Akt-S473, Akt, phospho-Stat5-Y694 (no. 9351), phospho-p44p42-T202/Y204-MAPK (P-Erk) (Cell Signaling); actin (Chemicon/Millipore); PCNA (Oncogene, Cambridge, MA, USA); PRLR (H-300), Ezrin (4A5), Erk1/2 (C-16/C14) Stat5 (C-17), Cyclin D1 (M-20), GST (B-14) (Santa Cruz Biotechnology, Santa Cruz, CA, USA); Elf5 (Thermo); Myc (Invitrogen); and FLAG (Sigma-Aldrich).

Statistics. All numerical data were examined for normal distribution. Parametric or non-parametric methods of one-way analysis of variance and Student's t-test were used, as appropriate, to analyze the differences between groups. Tukey's test was used for post hoc multiple comparison procedures. The log-rank test was used for the binary data on cumulative frequency of first pregnancy. Contingency tables of categorical data on litter sizes and pup weight at weaning ( $3 \times 4$ and $2 \times 3$ tables, respectively) were analyzed by $\chi^{2}$-test. For all tests, statistical significance was considered for $P<0.05$. Unless otherwise indicated, values are presented as means \pm S.E.M.

\section{Conflict of Interest}

The authors declare no conflict of interest.

Acknowledgements. We thank Yoko Takahashi and Theodora Georgescu for technical assistance. This work was supported by NCl-CA107201, American Recovery and Reinvestment Act supplement, and UTMDACC Bridge Funding to 
MMG. Animal breeding and tissue processing were partly supported by NCI-CA16672 and P30-DK56338 (funding the TMC Digestive Diseases Center), respectively.

1. Hennighausen L, Robinson GW. Information networks in the mammary gland. Nat Rev Mol Cell Biol 2005; 6: 715-725.

2. Bole-Feysot C, Goffin V, Edery M, Binart N, Kelly PA. Prolactin (PRL) and its receptor: actions, signal transduction pathways and phenotypes observed in $\mathrm{PRL}$ receptor knockout mice. Endocr Rev 1998; 19: 225-268.

3. Ormandy CJ, Naylor M, Harris J, Robertson F, Horseman ND, Lindeman GJ et al. Investigation of the transcriptional changes underlying functional defects in the mammary glands of prolactin receptor knockout mice. Recent Prog Horm Res 2003; 58 : 297-323.

4. Srivastava S, Matsuda M, Hou Z, Bailey JP, Kitazawa R, Herbst MP et al. Receptor activator of NF-kappaB ligand induction via Jak2 and Stat5a in mammary epithelial cells. J Biol Chem 2003; 278: 46171-46178.

5. Brockman JL, Schroeder MD, Schuler LA. PRL activates the cyclin D1 promoter via the Jak2/Stat pathway. Mol Endocrinol 2002; 16: 774-784.

6. Ormandy CJ, Camus A, Barra J, Damotte D, Lucas B, Buteau H et al. Null mutation of the prolactin receptor gene produces multiple reproductive defects in the mouse. Genes Dev 1997; 11: 167-178.

7. Shillingford JM, Miyoshi K, Robinson GW, Grimm SL, Rosen JM, Neubauer H et al. Jak2 is an essential tyrosine kinase involved in pregnancy-mediated development of mammary secretory epithelium. Mol Endocrinol 2002; 16: 563-570.

8. Wagner KU, Krempler A, Triplett AA, Qi Y, George NM, Zhu J et al. Impaired alveologenesis and maintenance of secretory mammary epithelial cells in Jak2 conditional knockout mice. Mol Cell Biol 2004; 24: 5510-5520.

9. Liu X, Robinson GW, Wagner KU, Garrett L, Wynshaw-Boris A, Hennighausen L. Stat5a is mandatory for adult mammary gland development and lactogenesis. Genes Dev 1997; 11: 179-186.

10. Fantl V, Stamp G, Andrews A, Rosewell I, Dickson C. Mice lacking cyclin D1 are small and show defects in eye and mammary gland development. Genes Dev 1995; 9: 2364-2372.

11. Sicinski P, Donaher JL, Parker SB, Li T, Fazeli A, Gardner H et al. Cyclin D1 provides a link between development and oncogenesis in the retina and breast. Cell 1995; 82: 621-630.

12. Bry C, Maass K, Miyoshi K, Willecke K, Ott T, Robinson GW et al. Loss of connexin 26 in mammary epithelium during early but not during late pregnancy results in unscheduled apoptosis and impaired development. Dev Biol 2004; 267: 418-429.

13. Fata JE, Kong YY, Li J, Sasaki T, Irie-Sasaki J, Moorehead RA et al. The osteoclast differentiation factor osteoprotegerin-ligand is essential for mammary gland development. Cell 2000; 103: 41-50.

14. Morales FC, Takahashi Y, Kreimann EL, Georgescu MM. Ezrin-radixin-moesin (ERM)binding phosphoprotein 50 organizes ERM proteins at the apical membrane of polarized epithelia. Proc Natl Acad Sci USA 2004; 101: 17705-17710.

15. Reczek D, Berryman M, Bretscher A. Identification of EBP50: a PDZ-containing phosphoprotein that associates with members of the ezrin-radixin-moesin family. J Cell Biol 1997; 139: 169-179.

16. Weinman EJ, Steplock D, Wang Y, Shenolikar S. Characterization of a protein cofactor that mediates protein kinase A regulation of the renal brush border membrane $\mathrm{Na}(+)-\mathrm{H}+$ exchanger. J Clin Invest 1995; 95: 2143-2149.

17. Hall RA, Ostedgaard LS, Premont RT, Blitzer JT, Rahman N, Welsh MJ et al. A C-terminal motif found in the beta2-adrenergic receptor, P2Y1 receptor and cystic fibrosis transmembrane conductance regulator determines binding to the $\mathrm{Na}+/ \mathrm{H}+$ exchanger regulatory factor family of PDZ proteins. Proc Natl Acad Sci USA 1998; 95: 8496-8501.

18. Georgescu MM, Morales FC, Molina JR, Hayashi Y. Roles of NHERF1/EBP50 in cancer. Curr Mol Med 2008; 8: 459-468

19. Ingraffea J, Reczek D, Bretscher A. Distinct cell type-specific expression of scaffolding proteins EBP50 and E3KARP: EBP50 is generally expressed with ezrin in specific epithelia, whereas E3KARP is not. Eur J Cell Biol 2002; 81: 61-68.

20. Stemmer-Rachamimov AO, Wiederhold T, Nielsen GP, James M, Pinney-Michalowski D, Roy JE et al. NHE-RF, a merlin-interacting protein, is primarily expressed in luminal epithelia, proliferative endometrium, and estrogen receptor-positive breast carcinomas. Am $J$ Pathol 2001; 158: 57-62.

21. Cardone RA, Bellizzi A, Busco G, Weinman EJ, Dell'Aquila ME, Casavola V et al. The NHERF1 PDZ2 domain regulates PKA-RhoA-p38-mediated NHE1 activation and invasion in breast tumor cells. Mol Biol Cell 2007; 18: 1768-1780.
22. Song J, Bai J, Yang W, Gabrielson EW, Chan DW, Zhang Z. Expression and clinicopathological significance of oestrogen-responsive ezrin-radixin-moesin-binding phosphoprotein 50 in breast cancer. Histopathology 2007; 51: 40-53.

23. Yun $\mathrm{CH}$, Oh S, Zizak M, Steplock D, Tsao S, Tse CM et al. cAMP-mediated inhibition of the epithelial brush border $\mathrm{Na}+/ \mathrm{H}+$ exchanger, $\mathrm{NHE} 3$, requires an associated regulatory protein. Proc Natl Acad Sci USA 1997; 94: 3010-3015.

24. Takahashi Y, Morales FC, Kreimann EL, Georgescu MM. PTEN tumor suppressor associates with NHERF proteins to attenuate PDGF receptor signaling. EMBO J 2006; 25: 910-920.

25. Kreimann EL, Morales FC, de Orbeta-Cruz J, Takahashi Y, Adams H, Liu TJ et al. Cortical stabilization of beta-catenin contributes to NHERF1/EBP50 tumor suppressor function. Oncogene 2007; 26: 5290-5299.

26. Pan $\mathrm{Y}$, Weinman EJ, Dai J. NHERF1 ( $\mathrm{Na}+/ \mathrm{H}+$ exchanger regulatory factor 1$)$ inhibits platelet-derived growth factor signaling in breast cancer cells. Breast Cancer Res 2008; 10: R5.

27. Camarillo IG, Thordarson G, Moffat JG, Van Horn KM, Binart N, Kelly PA et al. Prolactin receptor expression in the epithelia and stroma of the rat mammary gland. $J$ Endocrinol 2001; 171: 85-95.

28. Shenolikar S, Weinman EJ. NHERF: targeting and trafficking membrane proteins. Am J Physiol 2001; 280: F389-F395.

29. Shibata T, Chuma M, Kokubu A, Sakamoto M, Hirohashi S. EBP50, a beta-cateninassociating protein, enhances Wnt signaling and is over-expressed in hepatocellular carcinoma. Hepatology 2003; 38: 178-186.

30. Choi YS, Chakrabarti R, Escamilla-Hernandez R, Sinha S. Elf5 conditional knockout mice reveal its role as a master regulator in mammary alveolar development: failure of Stat5 activation and functional differentiation in the absence of Elf5. Dev Biol 2009; 329: 227-241.

31. Das R, Vonderhaar BK. Transduction of prolactin's (PRL) growth signal through both long and short forms of the PRL receptor. Mol Endocrinol 1995; 9: 1750-1759.

32. Hall RA, Premont RT, Chow CW, Blitzer JT, Pitcher JA, Claing A et al. The beta2adrenergic receptor interacts with the $\mathrm{Na}+/ \mathrm{H}+$-exchanger regulatory factor to control $\mathrm{Na}+/ \mathrm{H}+$ exchange. Nature 1998; 392: 626-630.

33. Li JG, Chen C, Liu-Chen LY. Ezrin-radixin-moesin-binding phosphoprotein-50/Na $+/ \mathrm{H}+$ exchanger regulatory factor (EBP50/NHERF) blocks U50,488H-induced down-regulation of the human kappa opioid receptor by enhancing its recycling rate. J Biol Chem 2002; 277: 27545-27552.

34. Boussadia O, Kutsch S, Hierholzer A, Delmas V, Kemler R. E-cadherin is a survival factor for the lactating mouse mammary gland. Mech Dev 2002; 115: 53-62.

35. Morales FC, Takahashi Y, Momin S, Adams H, Chen X, Georgescu MM. NHERF1/EBP50 head-to-tail intramolecular interaction masks association with PDZ domain ligands. Mol Cell Biol 2007; 27: 2527-2537.

36. Turunen $\mathrm{O}$, Wahlstrom $\mathrm{T}$, Vaheri A. Ezrin has a $\mathrm{COOH}$-terminal actin-binding site that is conserved in the ezrin protein family. J Cell Biol 1994; 126: 1445-1453.

37. Provost E, Rimm DL. Controversies at the cytoplasmic face of the cadherin-based adhesion complex. Curr Opin Cell Biol 1999; 11: 567-572.

38. Brdickova N, Brdicka T, Andera L, Spicka J, Angelisova P, Milgram SL et al. Interaction between two adapter proteins, PAG and EBP50: a possible link between membrane rafts and actin cytoskeleton. FEBS Lett 2001; 507: 133-136.

39. Park DS, Lee H, Frank PG, Razani B, Nguyen AV, Parlow AF et al. Caveolin-1-deficient mice show accelerated mammary gland development during pregnancy, premature lactation, and hyperactivation of the Jak-2/STAT5a signaling cascade. Mol Biol Cell 2002; 13: $3416-3430$

40. Dai JL, Wang L, Sahin AA, Broemeling LD, Schutte M, Pan Y. NHERF $(\mathrm{Na}+/ \mathrm{H}+$ exchanger regulatory factor) gene mutations in human breast cancer. Oncogene 2004; 23 8681-8687.

41. Smalley M, Ashworth A. Stem cells and breast cancer: a field in transit. Nat Rev Cancer 2003; 3: 832-844.

42. Hayashi Y, Molina JR, Hamilton SR, Georgescu MM. NHERF1/EBP50 is a new marker in colorectal cancer. Neoplasia 2010; 12: 1013-1022.

43. Molina JR, Agarwal NK, Morales FC, Hayashi Y, Aldape KD, Cote G et al. PTEN, NHERF1 and PHLPP form a tumor suppressor network that is disabled in glioblastoma. Oncogene 2012; 31: 1264-1274.

Cell Death and Disease is an open-access journal published by Nature Publishing Group. This work is licensed under the Creative Commons Attribution-NonCommercial-No Derivative Works 3.0 Unported License. To view a copy of this license, visit http://creativecommons.org/licenses/by-nc-nd/3.0/ 